Specifically Prescribed Dynamic

Thermodynamic Paths and

Resolidification Experiments

J. Nguyen, D. Orlikowski, F. Streitz, N. Holmes, J. Moriarty

November 25, 2003

Shock Compression of Condensed Matter 
This document was prepared as an account of work sponsored by an agency of the United States Government. Neither the United States Government nor the University of California nor any of their employees, makes any warranty, express or implied, or assumes any legal liability or responsibility for the accuracy, completeness, or usefulness of any information, apparatus, product, or process disclosed, or represents that its use would not infringe privately owned rights. Reference herein to any specific commercial product, process, or service by trade name, trademark, manufacturer, or otherwise, does not necessarily constitute or imply its endorsement, recommendation, or favoring by the United States Government or the University of California. The views and opinions of authors expressed herein do not necessarily state or reflect those of the United States Government or the University of California, and shall not be used for advertising or product endorsement purposes. 


\title{
SPECIFICALLY PRESCRIBED DYNAMIC THERMODYNAMIC PATHS AND RESOLIDIFICATION EXPERIMENTS
}

\author{
Jeffrey H. Nguyen*, Daniel Orlikowski*, Frederick H. Streitz*, Neil C. Holmes* and John A. \\ Moriarty* \\ *Physics and Advanced Technologies, Lawrence Livermore National Laboratory, Livermore CA 94550
}

\begin{abstract}
We describe here a series of dynamic compression experiments using impactors with specifically prescribed density profiles. Building upon previous impactor designs, we compose our functionally graded density impactors of materials whose densities vary from about $0.1 \mathrm{~g} / \mathrm{cc}$ to more than $15 \mathrm{~g} / \mathrm{cc}$. These impactors, whose density profiles are not restricted to be monotonic, can be used to generate prescribed thermodynamic paths in the targets. These paths include quasi-isentropes as well as combinations of shock, rarefraction, and quasi-isentropic compression waves. The time-scale of these experiments ranges from nanoseconds to several microseconds. Strain-rates in the quasi-isentropic compression experiments vary from approximately $10^{4} s^{-1}$ to $10^{6} s^{-1}$. We applied this quasi-isentropic compression technique to resolidify water where ice is at a higher temperature than the initial water sample. The particle velocity of quasiisentropically compressed water exhibits a two-wave structure and sample thickness scales consistently with water-ice phase transition time. Experiments on resolidification of molten bismuth are also promising.
\end{abstract}

\section{INTRODUCTION}

Traditionally, the extreme high-pressure phase diagram is studied through a few static and dynamic techniques: static compression involving diamond anvil cells (DAC) [1], shock compression [2, 3], and quasi-isentropic compression $[4,5,6,7,8,9,10]$. Static DAC experiments explore equilibrium material properties along an isotherm or an isobar [1]. Dynamic material properties can be explored with shock compression [2, 3], probing single states on the Hugoniot, or with quasi-isentropic compression [4, $5,6,7,8,9,10]$. In the case of shocks, pressures variation - as high as several megabars - typically occurs on a sub-nanosecond time scale or faster [11]. Previous quasi-isentropic techniques have yielded pressure ramps on the 10-100 nanosecond time-scale for samples that are several hundred microns thick [4, $5,6,7]$. In order to understand material response at high temperatures and high pressures, we need to span the dynamic range (strain rates, relaxation times, etc.) as well as control the thermodynamic path that the system experiences. Compression rates, for instance, need to bridge that of static experiments and those of Z-accelerator $\left(10^{6} s^{-1}\right)$ [4] and laser ab- lation $\left(10^{6} s^{-1}\right.$ to $\left.10^{8} s^{-1}\right)$ [7]. Here, we present a new technique which not only extends the compression time to several microseconds, but also extends the accessible states beyond the Hugoniot or room temperature adiabat. The strain rate in these experiments can vary from $10^{4}-10^{6} \mathrm{~s}^{-1}$, effictively bridging the gap between static compression and quasi-isentropic compression at the Z-accelerator [4] or using a laserablation drive [7].

We applied this new compression technique to resolidify water and molten bismuth. Unlike freezing, the final resolidified state is typically at higher temperature and pressure than the initial state. Rapid compressions such as shocks would impart excessive energy to the system to solidify the sample. Controlled compressions such as the quasi-isentropic compression technique described here can rapidly pressurize the sample across the melt curve without excessively raising the temperature of the system.

\section{EXPERIMENT}

Our targets are made of a sample tamped by a $10 \mathrm{~mm}$ $\mathrm{LiF}$ window to keep it at elevated pressures. The 

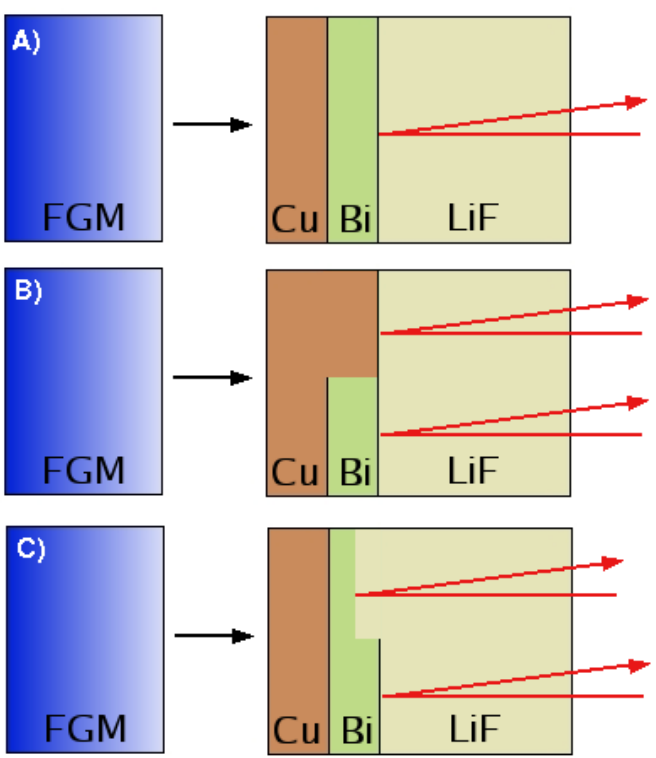

FIGURE 1. Experimental set-up: an FGM impactor designed with an increasing density (shock impedance) impacts a copper container that holds liquid bismuth. We measured particle velocity $U_{p}$ at the $\mathrm{Bi} / \mathrm{LiF}$ and $\mathrm{Cu} / \mathrm{LiF}$ interface using VISAR probes. (a) Single VISAR measurement of the particle velocity at the $\mathrm{Bi} / \mathrm{LiF}$ interface. (b) Dual VISAR measurements of the "witness" $\mathrm{Cu} / \mathrm{LiF}$ interface and the $\mathrm{Bi} / \mathrm{LiF}$ interface. (c) Dual VISAR measurements of two different Bi sample thicknesse. Water targets are similar, but with aluminum container.

$\mathrm{LiF}$ windows are coated with a few $\mu \mathrm{m}$ layer of silver for interface velocity measurement purposes. By measuring this sample/LiF interface velocity with a velocity interferometer (VISAR) [12], we can infer the pressure history at the interface. In the specifically prescribed dynamic compression experiments, we launch the functionally graded material (FGM) impactors against $\mathrm{Cu} / \mathrm{LiF}$ targets. The $\mathrm{Cu}$ plates (up to $5 \mathrm{~mm}$ thick) are flat ( $5 \mu \mathrm{m}$ tolerance) and are attached to a single crystal $\mathrm{LiF}$ window (more than $10 \mathrm{~mm}$ thick). Copper was chosen for its lack of a phase transition in the pressure-temperature region under study. For bismuth resolidification experiments, we used three different targets each comprised of a copper vessel, solid bismuth and an attached LiF window (Fig. 1). To liquify bismuth, the entire assembly is resistively heated to $577^{\circ} \mathrm{K}\left(30^{\circ} \mathrm{K}\right.$ above the melting point for $\mathrm{Bi}$ ) for a minimum of

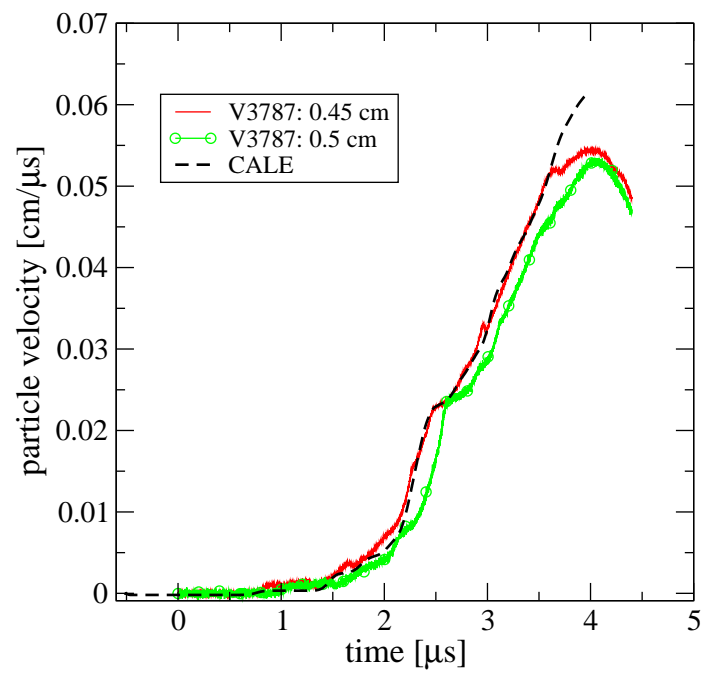

FIGURE 2. Typical compression light-gas gun experiment (impactor velocity $1.0 \mathrm{~km} / \mathrm{s}$ (V3787)). Simultaneous particle velocity measurements of two different $\mathrm{Cu}$ samples $(0.45 \mathrm{~cm}$ : red solid line; and $0.50 \mathrm{~cm}$ : green solid line). A hydrodynamic calculation (CALE) [14] that models the impactor composition, and simulates the experiment in one dimension. The deviation of the experiment from calculation at $0.05 \mathrm{~cm} / \mu$ s is due to rarefraction (release) waves from the side of the $\mathrm{Cu}$-target.

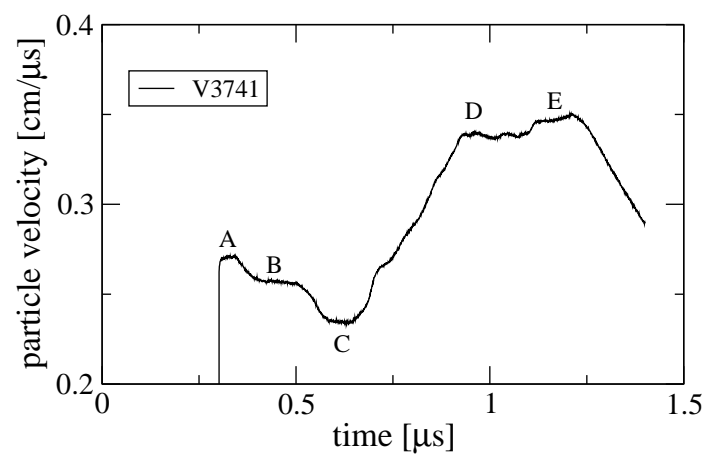

FIGURE 3. A VISAR trace produced through a complex density arrangement in the impactor shows that the $\mathrm{Cu}$ system was controlled and held through several thermodynamic states via a shock, controlled releases, and quasicompression.

fifteen minutes prior to impact, while a surrounding stainless steel armature supporting heat shields and the VISAR probes remain at near ambient temperature. Care was taken in preparing the bismuth sample since densification can occur upon melting 


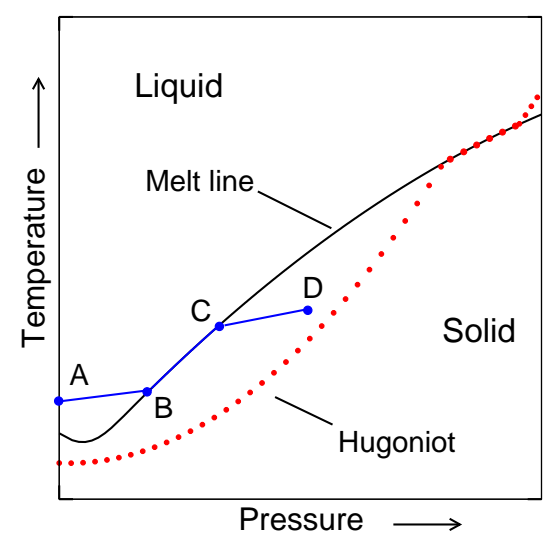

FIGURE 4. A schematic phase diagram of bismuth with an isentrope (ABCD) sketching the pathway to resolidification: a solid bismuth system is heated and melted to point $\mathrm{A}$; the liquid is then compressed along its isentrope(blue line) to point $\mathrm{B}$; the heat of fusion contained by the system is released as it undergoes a first-order phase transition until $\mathrm{C}$, from which the now solid bismuth is isentropically compressed to its final state at D. The principal Hugoniot (from ambient conditions) indicates approximately the boundary of the phase diagram previously explored (red dotted line). A schematic phase diagram of water is similar, but with the principal Hugoniot starting at A.

at ambient conditions. Bulk melting of bismuth was easily verified by the appearance of at $T_{\text {melt }}$ a distinctive plateau in temperature during heating. In the water resolidification experiments, aluminum is used as vessel material instead of copper for better impedance matching. The vacuum-pumped sample chamber is loaded with water a few minutes prior to shot. A thin silver coating is used to protect the $\mathrm{LiF}$ window from contact with water during the experiment.

Driving the specifically prescribed thermodynamic paths in the samples are our new FGM impactors. They are novel innovations, but are based on previous techniques $[9,10]$. We employed three different impactors, each one providing a specific density range. A complete impactor can be made using any one technique or any combinations thereof. The first of these is a functionally graded material impactor made of aluminum and tungsten powders in a resin matrix with densities ranging from $1.2 \mathrm{~g} / \mathrm{cc}$ to $8 \mathrm{~g} / \mathrm{cc}$. To achieve extremely low density, we adapted the graded density technique to a foam matrix $(0.1 \mathrm{~g} / \mathrm{cc}$ to $2.7 \mathrm{~g} / \mathrm{cc})$. This extends the lower density to $0.013 \mathrm{~g} / \mathrm{cc}$. However, we typically restricted the lowest density to about $0.1 \mathrm{~g} / \mathrm{cc}$, which still yields quasi-isentropic compression for impactor velocities of $4 \mathrm{~km} / \mathrm{s}$. The third part is comprised of sintered aluminum and tungsten powder $(2.7 \mathrm{~g} / \mathrm{cc}$ to $15 \mathrm{~g} / \mathrm{cc})$. We vary the density by manually adding layers with pre-mixed densities. The impactors typically have between 10 to 25 layers of $200 \pm 20 \mu \mathrm{m}$ thick. As our techniques improve, layer thicknesses continue to decrease. The variation in the shock impedance is gradual and specifically prescribed. Unlike the gravity assisted "pillow" technique $[9,10]$, we can within some limits construct the impactors with arbitrary variation in shock impedance profile (Figs. 2-3). This allows us to tailor thermodynamic paths from a simple quasiisentrope to any combination of shock, dynamic release, controlled release, and quasi-isentropic compression.

\section{COMPUTER SIMULATIONS}

To quantify the thermodynamic path for various compression experiments, we have developed analytic equation-of-states (EOS) for each of the layers $(\geq 20)$ comprising the impactor within the context of hydrodynamic production codes here at LLNL [14], where Steinberg-Guinan strength models [15] were used. The EOS's for impactor was developed during the experimental development of the impactor, while the EOS for the target material is well known. As demonstrated in fig. 2, the hydrodynamic simulations describe well the complete experimental assembly of impactor, target, and window.

\section{DYNAMIC COMPRESSION}

In Fig. 2, we present particle velocities $U_{p}$ of the $\mathrm{Cu}$ $\mathrm{LiF}$ interface, measured with a velocity interferometer (VISAR) [12]. The experiment was carried out simultaneously on two different samples with different thicknesses. By varying the velocity at which similar impactors are launched, we can also readily change 

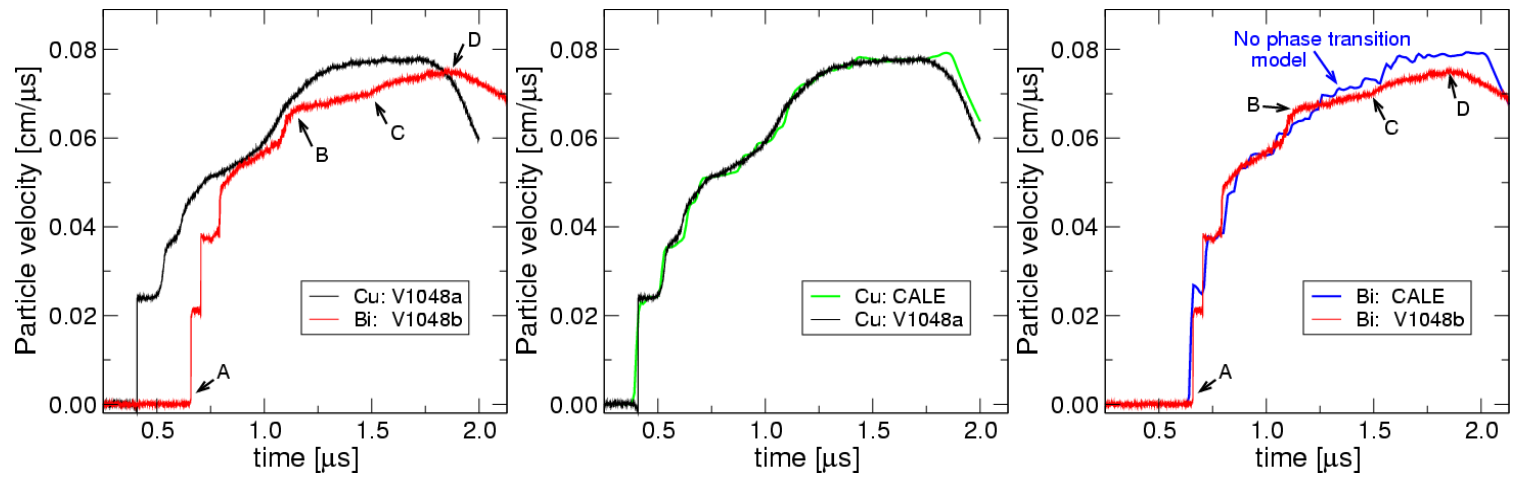

FIGURE 5. (a: left figure ) Particle velocities observed simultaneously using the dual VISAR setup (Fig. 1 (b)): data from the $\mathrm{Cu} / \mathrm{LiF}$ interface VISAR (black) indicates the applied pressure profile, while liquid $\mathrm{Bi} / \mathrm{LiF}$ interface VISAR (red) indicates the response of the $\mathrm{Bi}$. The ABCD points indicate that correspond to points sketched in Fig. 4. Peak pressure is obtained at point $\mathrm{D}$. The $\mathrm{Cu}$ sample is $1.75 \mathrm{~mm}$ thick, while the Bi sample is $0.87 \mathrm{~mm}$ thick; the impactor velocity is $1.3 \mathrm{~km} / \mathrm{s}$. The sudden flattening (B-C) in slope seen in the liquid particle velocity trace near $1.2 \mu$ s suggests the solidification transition. In (b: center figure) the $\mathrm{Cu} U_{p}$ from (a) is shown in conjunction with the hydrodynamic simulation modeling the impact on the $\mathrm{Cu}$. In (c: right figure) the $\mathrm{Bi} U_{p}$ is shown, where the same modeled impactor determined in (b) was used. The hydrodynamic simulation does not contain any phase transitions; therefore, the simulation shows the system's response as if there phase transition did not occur.

the compression rate of the sample. Conversely, one can also vary the impactor density profile to achieve different compression rates at the same shot velocity. Furthermore, the thermodynamic path is within numerical error of the adiabat for $\mathrm{Cu}$, but deviates from the Hugoniot. This deviation increases for high impactor velocities. The strain-rate for these experiments ranges from $10^{4} \mathrm{~s}^{-1}$ to $10^{6} \mathrm{~s}^{-1}$. These strainrates are slow enough for complete thermodynamic equilibrium to exist [16].

To utilize this impactor design more fully, we have manipulated the density profile of the impactor so as to drive the target sample through various thermodynamic states. As an initial example, we reversed the impactor used in figure 2 . This results in a strong shock followed by a rarefraction (release) wave- $\mathrm{a}$ decrease in $\mathrm{U}_{p}$ or pressure. However, the strain-rate of the release in this case is controlled to be slower than if the release wave $\left(5 \times 10^{10} \mathrm{~s}^{-1}\right)$ had propagated from the free surface of the backend of the impactor. Such impactor could be used to melt samples on release [17], but at a variable rate from fast to that commensurate with the rate of heat transport. It could also be used to simulate a Taylor wave. Manipulation of the density profile of the impactor can be much more complex, diversifying the thermodynamic paths available. In figure 3, the $\mathrm{Cu}$ is initially shocked to point $\mathrm{A}$, released to point $\mathrm{B}$, released still further to point $\mathrm{C}$, then compressed to point $\mathrm{D}$ and finally compressed one more time to point E, from which the system is allowed to release. At each point the system was held for some time. This VISAR trace is rather complex, as is the phase path and strain-rate effects. The movement and control of the effective pressure during the experiment allows the sample to completely reach mechanical equilibrium before other thermodynamic changes are induced; this possibly allows the examination of multiple phase transitions, where the sample equilibriates between phase transitions.

\section{RESOLIDIFICATION}

We initially investigated resolidification of molten bismuth during quasi-isentropic compression by measuring simultaneously (synchronous to within $20 \mathrm{~ns}$ ) the resulting particle velocities, which are proportional to pressure, from both a copper and liquid bismuth samples (Fig. 5b). The particle velocity measured from the $\mathrm{Bi} / \mathrm{LiF}$ interface indicates a later arrival of the first weak shock, which is consistent with the lower sound speed in liquid $\mathrm{Bi},(1.65 \mathrm{~km} / \mathrm{s}$ vs. $3.55 \mathrm{~km} / \mathrm{s}$ in $\mathrm{Cu})[18,19,20]$. After three weak shocks in the Bi sample, the particle velocity continues to smoothly increase with the applied pressure, 
exhibiting all the same characteristics seen in the copper trace until approximately $1.2 \mu \mathrm{s}$, at which point the slope of the liquid Bi particle velocity flattens while the copper velocity continues to increase, suggesting the onset of the phase transition (point B in Fig. 4). This behavior is similar to that seen with models for solid-solid transitions [21, 22, 16, 23]. As these hydrodynamic simulations do not include the effects of first-order phase transitions, deviations from the model for bismuth (Fig. 5 (c)), but not for copper (fig. 5 (b)) are expected. As the system moves along the melt line, the heat of fusionnormally released to the surrounding medium upon freezing - must be absorbed by the sample, since the heat transport by phonon modes is too slow to effectively reduce the temperature of the system within the time of the transition. This results in an increase in the sample temperature. With increasing temperature and pressure, the system-now with an growing percentage of solid bismuth-moves along the melt line (from B to $\mathrm{C}$ in Fig. 4) until just before $1.5 \mu \mathrm{s}$. At this time, the newly solidified Bi sample is driven off the melt curve and begins approaching its final compressed state (point $\mathrm{D}$ ). This is indicated by a change in particle velocity slope seen in the $\mathrm{Bi}$ sample at $1.48 \mu \mathrm{s}$, but absent in the $\mathrm{Cu}$ trace.

Using a different experimental setup (fig. 1c), we simultaneously measured two liquid Bi samples of different thicknesses. We then empirically calculated the Lagrangian sound speed [6] to directly verify the resolidification of the liquid Bi. Sound velocity in the solid phase is expected to be higher than that in the liquid phase at similar pressure due to the contribution of shear strength, which is non-existent in liquids. The sound velocity of liquid bismuth at ambient pressure (near the starting point of our experiments) is $1.65 \mathrm{~km} / \mathrm{s}[18,19,20]$ ) and for solid bcc-bismuth at approximately $0.1 \mathrm{Mbar}$ on the Hugoniot (near the end point of our compression path), the sound velocity is $2.9-3.1 \mathrm{~km} / \mathrm{s}[19,25]$, suggesting that we should observe a large change in sound velocity upon resolidification. In each experiment, we observe an increase in $C_{L}$ as the liquid is compressed, followed by a divergent region in $C_{L}$ as function of $U_{p}$ during the transition and finally a decay to a sound speed appropriate for a solid. While a divergence in the $C_{L}$ is expected based on solid-solid transformation within this Lagrangian analysis, the divergence here is contrary to expectation and can be related back to transient waves within the system, which limit the La-

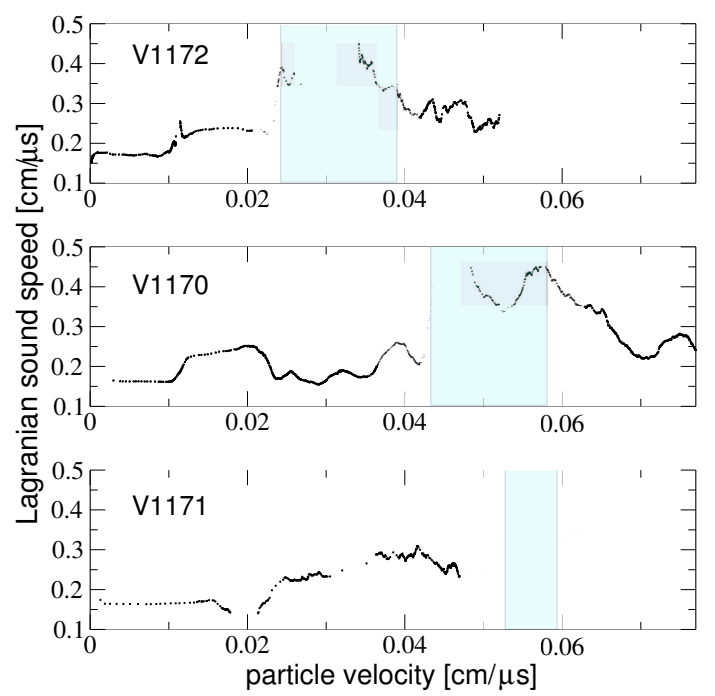

FIGURE 6. Empirically calculated Lagrangian sound velocity from experiments V1170-V1172. The blue shaded areas represent regions of particle velocity where the phase transition from liquid $\mathrm{Bi}$ to solid $\mathrm{Bi}$ is suspected. In this calculation, plateaus in the VISAR data were ignored, e.g. in V1171 near $0.5 \mu \mathrm{s}$.

grangian analysis. Furthermore, we cannot exclude the possibility that voids (caused by densification of bismuth on melting at ambient pressure) or impurities in the sample may have caused the divergence in sound velocities. Further investigations are needed.

We also explored resolidification in water using similar experimental set-up (fig. 7c), but with aluminum container. The particle velocities of the water/LiF interface (fig. 7) exhibit two wave structures indicative of phase transition. The water-ice transition times scale with water sample thickness as expected. (Very similar results were obtained in simulations of the $\alpha-\varepsilon$ transition in $\mathrm{Fe}$, for instance.) Initial simulations of this isentropic driven experiment indicate that the pressure associated $(\approx 2.2 \mathrm{GPa})$ with the plateau region $\left(U_{p} \approx 200 \mathrm{~m} / \mathrm{s}\right)$ are consistent with the $\mathrm{H}_{2} \mathrm{O}$ phase diagram; however, further simulations are required.

\section{CONCLUSION}

Our experiments and simulations indicate that a nearly-isentropic pressure wave can be produced in 


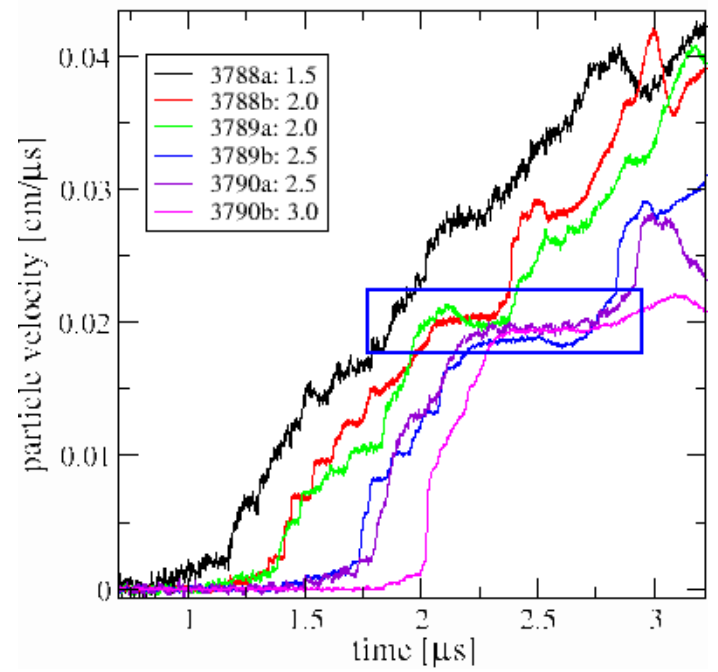

FIGURE 7. Particle velocities (VISAR) of water/LiF interface. Three sets of dual VISAR measurements of water/LiF interface. Water sample thicknesses are listed in the figure. The interface velocities exhibit two-wave structure indicative of phase transition at $\approx 200 \mathrm{~m} / \mathrm{s}$. The transition times scale with sample thicknesses.

a two-stage, light-gas gun (with a loading time ranging from ns to $\mu \mathrm{s}$ ) by using specifically prescribed density profile impactors. Additionally, with these impactors, we are able to shape the applied compression, and thereby dynamically probe those features of a phase diagram, which have some inherent time dependence, such as phase transitions and material response (strength properties, work hardening, fatigue?) along various thermodynamic paths. The hydrodynamic simulations have not only been used to model the experiments, but also have been used as a tool to design the density profile of the impactor for a desired experiment. By employing quasi-isentropic compression on molten bismuth and water, we found the two-phase wave structure signatures for resolidification. Additionally, the water sample thickness scales with resolidification time.

We would like to acknowledge useful discussions with R. Chau and R. Minich and the dedicated efforts of W. Hall N. Hinsey, M. O'Shell, E. Loughnane,J. van Lue, J. van Lewen, K. Lewis, L. Raper, K. Stickle, S. Weaver,and E. White. This work was performed under the auspices of the U.S.Department of Energy by University of California Lawrence Livermore National Laboratory under contract No. W-
7405-Eng-48.

\section{REFERENCES}

1. M. Eremets, High Pressure Experimental Methods, Oxford University Press, Oxford, 1996.

2. Y. B. Zel'dovich and Y. P. Raizer, Physics of Shock Waves and High-Temperature Hydrodynamic Phenomena. (Academic Press, New York, 1967).

3. G. E. Duvall and R. A. Graham, Rev. Mod. Phys. 49, 523 (1977).

4. C. A. Hall, Phys. Plasma 7, 2069 (2000).

5. C. A. Hall et al., Rev. Sci. Instru. 72, 3587 (2001).

6. D. B. Reisman et al., J. Appl. Phys. 89, 1625 (2001).

7. J. Edwards, Phys. Rev. Lett. (submitted) (2003).

8. Q. Shen et al., Acta Phys. Sinica, 51, 1759 (2002).

9. L. M. Barker, High-pressure Quasi-isentropic Impact Experiments. Shock Waves Cond. Matt. J. R. Asay, R. A. Graham and G. K. Straub, (eds.) (Elsevier Sci. Pub., Amsterdam)(1984).

10. L. C. Chhabildas, J. R. Asay \& L. M. Barker, Shear Strength of Tungsten Under Shock- and QuasiIsentropic Loading to $250 \mathrm{GPa}$, SAND88-0306 Sandia Nat. Lab., Albuquerque, New Mexico (April 1988).

11. W. G. Hoover, Phys. Rev. Lett.42, 1531 (1979).

12. L. M. Barker and R. E. Hollenbach, J. Appl. Phys. 43, 4669 (1972).

13. W. J. Nellis, S. T. Weir, and A. C. Mitchell, Phys. Rev. B 59, 3434 (1999).

14. R. Tipton, R. Managan, and P. Amala, CALE Users Manual (Lawrence Livermore National Laboratory, Livermore, California)(2002).

15. D. J. Steinberg, S. G. Cochran, and M. W. Guinan, J. Appl. Phys. 51, 1498 (1980); D. J. Steinberg and C. M. Lund, J. Appl. Phys. 65, 1528 (1989).

16. D. C. Wallace, Phys. Rev. B 22, 1477 (1980).

17. C. Mabire and P. L. Héreil, Shock Comp. Cond. Matt. M. D. Furnish, L. C. Chhabildas, and R. S. Hixson, (eds.) (AIP Conf. Proc., Melville, New York)(1999) pp. 93-96.

18. E. G. Pashuk, \& B. P. Pashaev, High Temp. 18, 256-260 (1980).

19. J. .L Péllisier \& N. Wetta, Physica A 289, 459-478 (2001).

20. N. Wetta \& J. L. Péllisier, Physica A 289, 479-497 (2001).

21. D. J. Andrews, J. Comput. Phys. 7, 310 (1971).

22. D. B. Hayes, J. Appl. Phys. 46, 3438 (1975).

23. K. Kadau, T. C. Germann, P. S. Lomdahl, B. L. Holian, Science 296, 1681 (2002).

24. D. J. Steinberg \& C. M. Lund, J. Appl. Phys. 65, 1528-1533 (1989).

25. M. N. Pavlovskii \& V. V. Komissarov, Sov. Phys. JETP 56, 1244-1246 (1982). 